\title{
GAIA Level 2 Morbidly Adherent Placentation
}

National Cancer Institute

\section{Source}

National Cancer Institute. GAIA Level 2 Morbidly Adherent Placentation. NCI Thesaurus.

Code C128745.

GAIA Level 2 Morbidly Adherent Placentation is defined by two criteria: first, ultrasound evidence of placenta previa; second, hypervascularity of the lower uterine segment, diagnosed during a laparotomy. Alternatively, Level 2 may be defined as difficulty with placental separation after delivery of the infant, during either a vaginal or a cesarean delivery, with resultant hemorrhage due to partial separation. 